\title{
Erratum to: Experimental Investigation on a Single NACA Airfoil's Nonlinear Aeroelasticity in Wake Induced Vibrations
}

\author{
Hairun Xie ${ }^{a}$, Yadong Wu ${ }^{a, *}$, Hua Ouyang ${ }^{a}$, and Anjenq Wang ${ }^{a}$ \\ ${ }^{a}$ School of Mechanical Engineering, Shanghai Jiao Tong University, Shanghai, 200240 China \\ *e-mail: yadongwu@sjtu.edu.cn \\ Received August 30, 2019
}

DOI: $10.1134 / \mathrm{S} 001546281910001 \mathrm{X}$

The list of the authors should read as follows:

\author{
Hairun Xie ${ }^{a}$, Yadong Wu ${ }^{a,{ }^{*}}$, Hua Ouyang ${ }^{a}$, and Anjenq Wang ${ }^{a}$ \\ ${ }^{a}$ School of Mechanical Engineering, Shanghai Jiao Tong University, Shanghai, 200240 China \\ *e-mail: yadongwu@sjtu.edu.cn
}

The original article can be found online at

https://doi.org/10.1134/S0015462819030121 